\title{
Chemoprevention of pancreatic cancer using solid-lipid nanoparticulate delivery of a novel aspirin, curcumin and sulforaphane drug combination regimen
}

\author{
DHRUVITKUMAR SUTARIA ${ }^{1,2}$, BALAGANGADHAR KARTHIK GRANDHI ${ }^{1}$, \\ ARVIND THAKKAR ${ }^{1}$, JEFFREY WANG ${ }^{1}$ and SUNIL PRABHU ${ }^{1}$ \\ ${ }^{1}$ Department of Pharmaceutical Sciences, College of Pharmacy, Western University of Health Sciences, Pomona, \\ CA 91766; ${ }^{2}$ College of Pharmacy, The Ohio State University, Columbus, OH 43210, USA
}

Received July 7, 2012; Accepted August 16, 2012

DOI: $10.3892 /$ ijo.2012.1636

\begin{abstract}
Pancreatic cancer is the fourth largest cause of cancer deaths in the Unites States and the prognosis is grim with $<5 \%$ survival chances upon diagnosis. The objective of this study was to assess the combined chemopreventive effect of solid lipid nanoparticle (SLN) encapsulated drugs aspirin (ASP), curcumin (CUR) and free sulforaphane (SFN) for the chemoprevention of pancreatic cancer. Experiments were carried out (1) to evaluate the feasibility of encapsulation of these chemopreventive agents within solid lipid systems and (2) to measure the synergistic effects of a combination of ASP with CUR in SLNs mixed with free SFN against cell proliferation and apoptosis in pancreatic cancer cells, MIA PaCa-2 and Panc-1. The SLNs were prepared using a modified solvent evaporation technique and were characterized for particle sizing, encapsulation efficiency and drug release. ASP and CUR SLNs were formulated within the particle size range of $150-250 \mathrm{~nm}$ and were found to have an encapsulation efficiency of 85 and $69 \%$, respectively. Sustained release of drugs over a $96 \mathrm{~h}$ period from SLNs was observed. The SLNs were stable over a 3-month storage period at room temperature. Cell viability studies demonstrated that combinations of low doses of ASP SLN $(25 \mu \mathrm{M})$, CUR SLN $(2.5 \mu \mathrm{M})$ and free SFN $(5 \mu \mathrm{M})$ significantly reduced cell viability by 43.6 and $48.49 \%$ in MIAPaca-2 and Panc-1 cell lines, respectively. Furthermore, increased apoptosis of 61.3 and $60.37 \%$ was found in MIA Paca- 2 and Panc- 1 cell lines, respectively, in comparison to the individual doses administered. Synergistic effects were demonstrated using MTS and apoptosis assays. Thus, this study successfully demonstrated the feasibility of using a solid lipid nanoparticulate system for the first time to deliver this novel
\end{abstract}

Correspondence to: Dr Sunil Prabhu, Department of Pharmaceutical Sciences, College of Pharmacy, Western University of Health Sciences, 309, East 2nd Street, Pomona, CA 91766, USA

E-mail: sprabhu@westernu.edu

Key words: chemoprevention, pancreatic cancer, combination therapy, nanotechnology, solid lipid nanoparticles combination chemoprevention regimen, providing valuable evidence for the usability of nanotechnology-based drug regimens towards pancreatic cancer chemoprevention.

\section{Introduction}

Pancreatic cancer is the fourth most common cause of cancer deaths in United States with a five-year survival rate of less than 5\%. According to American Cancer Society, in the US alone it is estimated that 43,920 individuals will be diagnosed with and 37,390 of them will die of pancreatic cancer in 2012 (1). Pancreatic cancer arises from the morphologically and genetically clearly defined precursor lesions through a step-wise accumulation of genetic alterations. In majority of the patients diagnosed with pancreatic cancer, symptoms do not develop until it is either unresectable or metastatic, rendering it difficult to cure (2). The low survival rate of patients points towards an increased need for novel strategies to combat pancreatic cancer. The concept of chemoprevention has recently received significant attention as a novel strategy towards preventing pancreatic cancer before it occurs (3). Some chemopreventive agents such as COX-2 inhibitors, green and black tea derivatives, $\beta$-carotene, vitamins, isothiocyanates, and farnesyl transferase inhibitors have been reported as potential chemopreventive agents (4-7).

Aspirin (ASP), a well-known non-steroidal antiinflammatory drug (NSAID), has emerged as a promising chemopreventive agent against various types of cancer. ASP is capable of suppressing pancreatic cancer growth both in vitro and in vivo (8). Cyclooxygenase-2 (Cox-2) enzyme which plays a key role in prostaglandin E2 (PGE2) synthesis is overexpressed in several cancers including pancreatic cancer. ASP has been shown to inhibit pancreatic carcinogenesis by the inhibition of Cox-2 enzymes (6). Studies associated with the use of ASP for pancreatic cancer chemoprevention have met with mixed results thus far (5,7). Given these conflicting reports on the use of ASP in pancreatic cancer, it reaffirms the need for further study of this drug in pancreatic cancer chemoprevention.

Curcumin (CUR), a diferuloylmethane is a derivative of spice turmeric (Curcuma longa). CUR has shown to have pronounced chemopreventive, anti-inflammatory, anti-oxidative 
and anti-carcinogenic activities in different cancer cell lines and murine cancer models (9-11). CUR has been shown to suppress $\mathrm{NF}-\kappa \mathrm{B}$ activation and NF- $\kappa \mathrm{B}$ gene products (12) and can induce p53-dependent apoptosis by induction of p53 in certain cancer cell lines (13). In pancreatic cancer cell lines, CUR acts by suppressing the activation of $\mathrm{NF}-\kappa \mathrm{B}$ through the inhibition of $\mathrm{I} \kappa \mathrm{B} \alpha$ protein (14).

Sulforaphane (SFN) is a naturally-occurring sulfurcontaining isothiocyanate found in cruciferous vegetables such as broccoli, brussel sprouts, cauliflower and cabbage (15). SFN has been shown to reduce NF- $\kappa \mathrm{B}$ activity and affect expression of NF- $\kappa \mathrm{B}$ mediated genes encoding adhesion molecules, inflammatory cytokines, growth factors and anti-apoptotic factors (16). SFN also modulates multiple targets, which regulate many cellular activities including oxidative stress, apoptosis induction, cell cycle arrest, angiogenesis and metastasis suppression (17).

There is an increasing interest to use a combination of chemopreventive agents that differ in their mode of action and target multiple pathways. This approach provides a means of obtaining low-dose therapy and increased efficacy with less toxicity $(18,19)$. It is noteworthy that the phase III clinical trial of difluoromethylornithine combination with sulindac has shown greater chemopreventive efficacy (19), which pave the way for the use of combinatorial regimens to achieve a synergistic effect. To date, no group has investigated the combined therapeutic effects of low dose mixtures of ASP, CUR and SFN on prevention of pancreatic cancer. Since all of these agents have different mechanisms of action, an anticipated synergistic effect in pancreatic cancer would provide valuable information in assessing combination chemopreventive regimens for eventual clinical use.

The method of delivery plays an important role in improving the overall drug bioavailability. Novel modes of delivery methods using microspheres and nanosphere technology are receiving wide attention as these have shown superior delivery compared to conventional dosage forms $(20,21)$. The strength of the drug delivery system is their ability to alter the pharmacokinetics and biodistribution of the drug. Nanoparticles have unusual properties that can be used to improve drug delivery. Provided they are within the nanometer size range, there is an increased uptake by the cells through enhanced permeation and retention (EPR) effect thereby making it a potential tool to diagnose and treat cancer. Nanosized drug delivery systems offer several advantages over the conventional delivery system such as controlled and sustained release of drugs, ability of the drug to cross the mucosal barriers, decreased renal and hepatic clearance, decreased immune recognition, increased half-lives of drugs due to slow and controlled release from the nanoparticulate systems, increased stability and solubility (22). All these advantages suggest the emerging role of nanoparticles in cancer therapy and chemoprevention demonstrating a need for further research in this area.

A nanotechnology-based drug delivery system, solid-lipid nanoparticles (SLN) has received considerable attention in the last few years as a convenient method of delivering drugs into the body in a controlled release manner (23). SLNs are commonly defined as solid nanoscaled lipid matrices in size range of 50-1,000 nm typically consisting of a solid lipid compound, surfactant and incorporated active ingredients. Additionally,
SLNs are biocompatible and act by protecting incorporated compounds against chemical degradation. However, the most important advantage of SLNs is that they increase the bioavailability of lipophilic drugs administered by the oral route (24). There is considerable evidence that SLNs act by carrying most of the drugs through the lymphatic system, and in part through the general blood circulation thus avoiding first pass metabolism (25). This allows for administration of lower doses with less chances of toxic effects, while maintaining efficacy. It has been observed that the formulations exhibited superior and better cytotoxicity profile compared to their corresponding free drug (26). Thus, the SLN delivery system due to the favorable physicochemical characteristics, controlled release kinetics would be ideal for delivery of lipophilic drugs like ASP, CUR and SFN.

Although many studies have been conducted to test the chemopreventive efficacy of ASP, CUR and SFN, no studies have been reported on the combined chemopreventive efficacy of these SLN encapsulated drugs. We recently demonstrated significant chemoprevention of colon cancer in rats using an orally administered drug loaded polylactide-co-glycolide (PLGA) polymer in a nanotechnology-based targeted delivery system (26). Here, we are proposing the use of a novel solidlipid nanoparticle (SLN) technology for the oral delivery of combinations of chemopreventive agents for pancreatic cancer. Thus, in the present study we formulate the above mentioned chemopreventive agents into SLNs and further evaluate their combined chemopreventive efficacy in two different human pancreatic cancer cells, MIA PaCa-2 and Panc-1.

\section{Materials and methods}

Materials. For the cell culture assays and solid lipid nanoparticle (SLN) formulations, the drugs ASP, CUR and SFN were obtained from LKT Laboratories (St. Paul, MN). Dimethyl sulfoxide (DMSO) was obtained from Sigma Chemicals (St. Louis, MO). Stearic acid (lipid) and Poloxamer 188 (emulsion stabilizer) was obtained from Spectrum Chemicals (Garden, CA). Dichloromethane (DCM) was obtained from Fisher Scientific (Houston, TX).

Human cell lines. Human pancreatic cancer cell lines MIA PaCa-2 and Panc-1 were obtained from ATCC (Rockville, MD). Cells were maintained in Dulbecco's modified Eagle's medium (DMEM) containing 10\% fetal bovine serum (FBS) obtained from ATCC. Cells were cultured at $37^{\circ} \mathrm{C}$ in a humidified atmosphere of $5 \% \mathrm{CO}_{2}$ and $95 \%$ air.

Preparation of solid lipid nanoparticles (SLNS). ASP and CUR SLNs were prepared using a hot melt oil-in-water (o/w) emulsion technique. Stearic acid was used as the solid lipid to make the nanoparticle formulations. Briefly, $1 \mathrm{mg}$ of stearic acid was melted by heating in a water bath at $70-80^{\circ} \mathrm{C}$. The drug $(100 \mathrm{mg})$ was suspended in $3 \mathrm{ml}$ of DCM. The suspended drug solution was then added to the melted stearic acid and heated until all DCM was evaporated. The water phase consisted of $1 \%$ poloxamer solution which was heated to the same temperature as that of the oil phase. The oil phase was then added to the poloxamer solution and the mixture was further sonicated for $5 \mathrm{~min}$ using an ultra-sonicator (Branson, Los Angeles, CA) to create an o/w 
emulsion. The emulsion so formed was then cooled and washed with water to remove excess free drug from the particle surface. SLNs were freeze-dried in a freeze dryer (Labconco, Kansas City, MO) and subjected to particle sizing and encapsulation efficiency determination.

Particle size measurement. The particle sizes of the formulated SLNs were measured using Nicomp submicron particle size analyzer Model 370 (New York, USA). Briefly, $5 \mathrm{mg}$ of the SLN formulation was suspended in $10 \mathrm{ml}$ of phosphate saline buffer (PBS, pH 7.4) and was sonicated for 2 min. Particle size was measured using $1 \mathrm{ml}$ of the suspension.

Encapsulation efficiency (\%) determination. Encapsulation efficiency (E.E) was determined by dissolving $10 \mathrm{mg}$ of the SLN formulation in DCM which dissolves the stearic acid and releases the drug entrapped within the lipid. DCM was evaporated under a current of inert air for $1 \mathrm{~h}$. Evaporation of DCM left a residue of the drug and lipid sticking to the bottom of the test tube. Drug was separated from the lipid by dissolving it in $5 \mathrm{ml}$ of acetonitrile. The drug was allowed to dissolve freely for about $30 \mathrm{~min}$ in a bath sonicator after which it was filtered through a $0.45 \mu \mathrm{m}$ filter. The resulting solution was further diluted to $20 \mathrm{ml}$ by adding acetonitrile. A total of $1 \mathrm{ml}$ of the resulting mixture was analyzed using Shimadzu LC-20 binary HPLC system (Columbia, MD). A total of $20 \mu \mathrm{l}$ of naproxen was used as an internal standard. E.E was calculated using the following expression: E.E $(\%)=$ amount $(\mathrm{mg})$ of drug per HPLC $\mathrm{method} /$ theoretical yield $(\mathrm{mg}) \times 100$.

Determination of drug release from SLNs. The in vitro release profile of ASP and CUR from SLNs was determined using a dialysis bag method. A hydrophilic dialysis membrane pouch (MWCO $12 \mathrm{kDa}$ ) served as the donor compartment. SLNs containing $100 \mathrm{mg}$ of the drug were suspended in $5 \mathrm{ml}$ of PBS and placed inside the membrane pouch. Subsequently, the pouch was lowered into a container with $100 \mathrm{ml}$ PBS solution containing $1 \%$ sodium lauryl sulfate (SLS) serving as the receptor medium for the study. At fixed time intervals, $5 \mathrm{ml}$ receptor medium was withdrawn and replaced with $5 \mathrm{ml}$ fresh medium. Samples were analyzed using HPLC. All the samples were carried out in triplicates.

Drug encapsulated SLN stability studies. Storage stability studies were conducted on ASP and CUR SLN formulations over a period of 3 months. Samples were stored at three different temperatures $4^{\circ} \mathrm{C}, 24^{\circ} \mathrm{C}$ and $37^{\circ} \mathrm{C}$ in closed glass vials. Particle size and encapsulation efficiencies were determined as indicators of storage stability of the prepared SLNs. All the studies were conducted in triplicate.

Cell viability (MTS) assay. The cell viability assay was performed according to manufacturer's protocol included with the Promega CellTitre 96 Aqueous MTS reagent (Madison, WI). Briefly, $2.5 \times 10^{3}$ cells of MIA PaCa- 2 cells and $4 \times 10^{3}$ cells of Panc-1 were seeded in 96-well plates and incubated for $24 \mathrm{~h}$. Test compounds ASP SLNs, CUR SLNs and free SFN alone and in combination at a concentration of $25 \mu \mathrm{M}, 2.5 \mu \mathrm{M}$ and $5 \mu \mathrm{M}$ respectively, were added and incubated for a period of $72 \mathrm{~h}$. On the last day of the incubation period, the growth medium
Table I. Particle size and encapsulation efficiency of drugloaded solid lipid nanoparticles.

\begin{tabular}{lcc}
\hline Drug & $\begin{array}{c}\text { Particle } \\
\text { size }(\mathrm{nm})\end{array}$ & $\begin{array}{c}\text { Encapsulation } \\
\text { efficiency }(\%)\end{array}$ \\
\hline Aspirin & $150 \pm 63$ & $85 \pm 5.2$ \\
Curcumin & $249 \pm 65$ & $69 \pm 3.0$ \\
\hline
\end{tabular}

was removed followed by addition of $100 \mu 1$ mixture consisting of $20 \%$ MTS and $1 \%$ of phenazine methosulfate (PMS) to the serum-free culture medium and incubated for $2 \mathrm{~h}$ at $37^{\circ} \mathrm{C}$. MTS is bioreduced by cells into formazan which can be measured at $490 \mathrm{~nm}$. Thus, the quantity of formazan product measured by the amount of $490 \mathrm{~nm}$ absorbance is directly proportional to the number of living cells in culture. $\mathrm{IC}_{50}$ values were determined using Prism software (San Diego, CA). All the samples were performed in triplicates. Each experiment was performed at least thrice.

Annexin V-PI apoptosis assay. The assay was performed according to manufacturer's protocol included with the Annexin V-fluorescein isothiocyanate (FITC) Vybrant Apoptosis assay kit \#3 ( Invitrogen, Green Island, NY). Briefly, $3 \times 10^{5}$ MIA PaCa-2 and Panc-1 cells were seeded in 6-well plates and incubated for a period of $24 \mathrm{~h}$. Test compounds ASP SLNs, CUR SLNs and free SFN alone and in combination at a concentration of $25 \mu \mathrm{M}, 2.5 \mu \mathrm{M}$ and $5 \mu \mathrm{M}$, respectively, were added and incubated for a period of $48 \mathrm{~h}$. After the incubation period, cells were washed twice with cold PBS (phosphate buffered saline) and then resuspended in $1 \mathrm{X}$ Annexin binding buffer such that the cell density was equivalent to $1 \times 10^{6}$ cells $/ \mathrm{ml}$. A total of $100 \mu \mathrm{l}$ of this cell suspension was then subjected to $5 \mu \mathrm{l}$ of FITC Annexin V and $1 \mu \mathrm{l}$ of the $100 \mu \mathrm{g} / \mathrm{ml}$ PI followed by incubation in the dark for $15 \mathrm{~min}$. After the incubation period, $400 \mu \mathrm{l}$ of $1 \mathrm{X}$ annexin binding buffer was added to the cells followed by gentle vortexing. The samples were kept on ice and were analyzed using Beckman Coulter Cytomics FC500 (Brea, CA). The fluorescence emission was measured at $530 \mathrm{~nm}$ (e.g. FL1) and >575 nm (e.g. FL3).

Statistical analysis. Results were expressed as mean \pm SEM. A one-way ANOVA followed by Tukey's post hoc analysis using Graph pad prism software (La Jolla, CA) was done to analyze and compare the results. A probability value of $\leq 0.05 \%$ was considered significant.

\section{Results}

Particle sizing of drug encapsulated SLNs. Nicomp volume weight was used as the standard measure for poly distribution type particle sizing. The SLNs of ASP and CUR were retained within the nanometer range (Table I). The particle size of ASP SLNs $(150 \mathrm{~nm})$ was lower than that of CUR $(249 \mathrm{~nm})$. All the SLNs showed optimal particle size with low variability.

Encapsulation efficiency (\%). The SLNs exhibited 85 and $69 \%$ encapsulation efficiency of the ASP and CUR, respectively, 


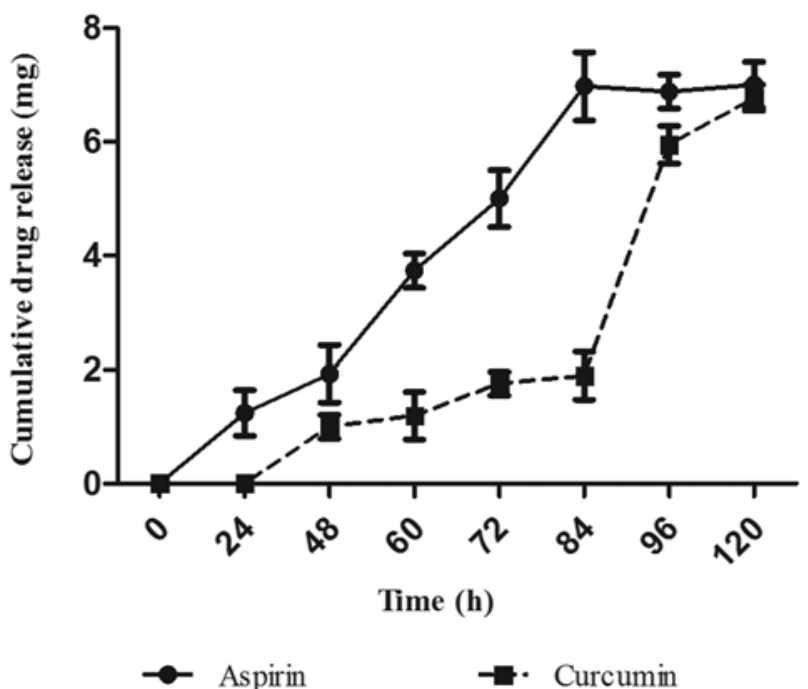

Figure 1. The release of aspirin and curcumin from solid lipid nanoparticles over a period of 5 days. The drug release was determined using a dialysis bag method and analysis of samples was done using an HPLC system. The data were plotted as mean \pm SEM. All the samples were carried out in triplicates.

within the lipid (Table I). The encapsulation efficiency was higher for ASP in comparison to CUR.

Drug release from the SLNS. The drug release studies were performed using membrane compartmental analysis. The release of the drug from nanoparticles prepared using stearic acid as lipid was conducted over a period of 5 days. As shown in Fig. 1, the release of ASP was faster compared to CUR. Release of CUR was not observed until a period of $24 \mathrm{~h}$. A cumulative drug release of approximately $7 \mathrm{mg}$ of ASP was observed within $90 \mathrm{~h}$ of the study showing faster release pattern in comparison to the curcumin SLNs. However, in comparison to ASP SLNs, CUR SLNs showed a slower drug release time profile releasing approximately $6.5 \mathrm{mg}$ of the drug entrapped. Both the formulations exhibited slow sustained release of the drug.
Drug encapsulated SLN stability studies. i) Particle size (nm). Particle size of ASP SLNs measured at the start of the study showed a size range of $160 \pm 32 \mathrm{~nm}$. After a three month duration, where samples were stored at three different temperatures $\left(4^{\circ} \mathrm{C}, 24^{\circ} \mathrm{C}\right.$ and $\left.37^{\circ} \mathrm{C}\right)$ the particle sizes were 165,164 and $180 \mathrm{~nm}$, respectively. For CUR SLNs, the size range at the start of the storage study was $250 \pm 40 \mathrm{~nm}$. At the end of 3 months study, the particle sizes were 260, 255 and $325 \mathrm{~nm}$, respectively. Thus both ASP and CUR SLNs exhibited stability at lower temperatures $\left(4^{\circ} \mathrm{C}\right.$ and $\left.24^{\circ} \mathrm{C}\right)$. However, some agglomeration was evident at $37^{\circ} \mathrm{C}$ which demonstrated an increase in particle size at that temperature (Fig. 2A). ii) Encapsulation efficiency (\%). ASP SLNs showed an initial encapsulation efficiency (E.E) of $85 \%$ at the start of the storage stability test. After 3 months at different temperatures, the E.E was found to be $82 \%\left(4^{\circ} \mathrm{C}\right)$, $80 \%\left(24^{\circ} \mathrm{C}\right)$ and $45 \%\left(37^{\circ} \mathrm{C}\right)$. The encapsulation efficiency of CUR SLNs was demonstrated to be $69 \%$ but after 3 months at different temperatures showed $70 \%\left(4^{\circ} \mathrm{C}\right), 65 \%\left(24^{\circ} \mathrm{C}\right)$ and $34 \%$ $\left(37^{\circ} \mathrm{C}\right)$ encapsulation (Fig. 2B). Thus, the encapsulation seemed to be affected at higher temperatures. Results of these studies demonstrated that storage at higher temperatures of $37^{\circ} \mathrm{C}$ could result in particle size and encapsulation changes which would have a direct adverse impact on the drug release characteristics from the SLNs used in the study.

$I C_{50}$ comparisons between free drugs and drug encapsulated SLNs. In order to study the effects of SLNs, the inhibitory concentrations $\left(\mathrm{IC}_{50}\right)$ values of the free drug and the drug encapsulated SLNs were compared. It was observed that the SLNs exhibited lower $\mathrm{IC}_{50}$ values in comparison to the free drug. In case of MIA PaCa- 2 cells, free ASP exhibited $\mathrm{IC}_{50}$ value of $2.6 \mathrm{mM}$. However, in case of drug encapsulated SLNs, the $\mathrm{IC}_{50}$ value was significantly reduced to $66.08 \mu \mathrm{M}$, showing approximately 38 -fold reduction compared to the free form of the drug (Fig. 3A). Whereas, free CUR exhibited the $\mathrm{IC}_{50}$ value of $19.6 \mu \mathrm{M}$ whereas CUR SLNs exhibited $\mathrm{IC}_{50}$ value of $4.93 \mu \mathrm{M}$ with $\sim 3$-fold reductions from its free form (Fig. 3B). Similarly for Panc-1 cells, the $\mathrm{IC}_{50}$ values obtained for free ASP and ASP SLNs were $2.4 \mathrm{mM}$ and $99.11 \mu \mathrm{M}$, respectively (Fig. 3C). Whereas, the $\mathrm{IC}_{50}$ values of CUR and CUR SLNs obtained
A

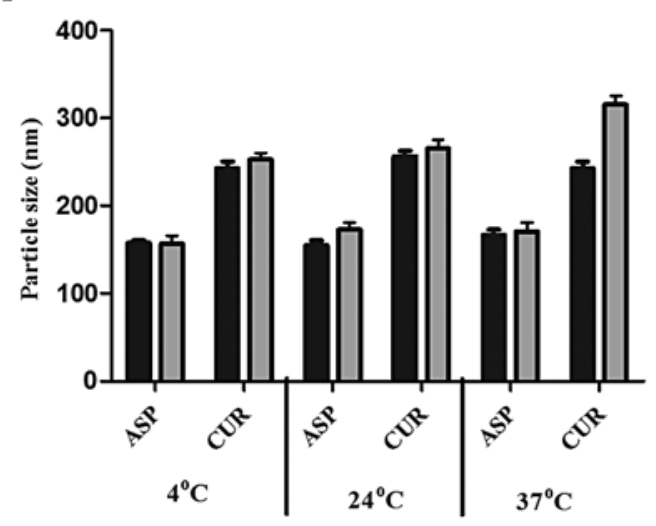

B

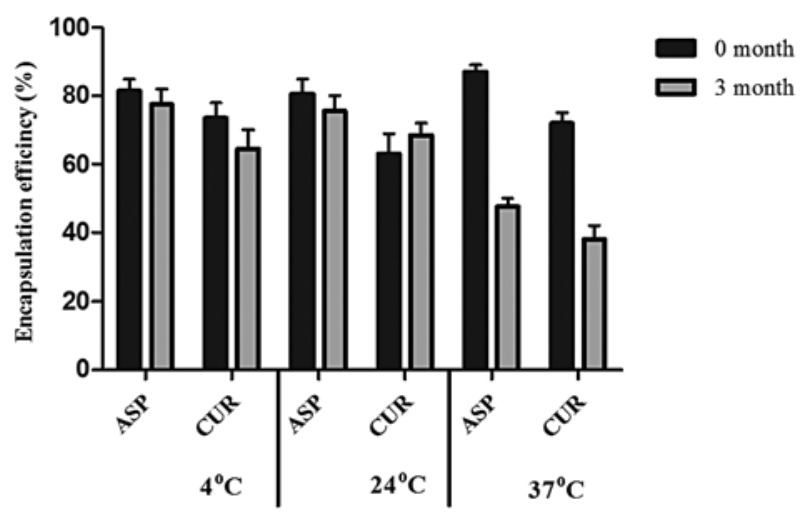

Figure 2. Storage stability data of aspirin (ASP) and curcumin (CUR) SLNs over a three month period at three different temperatures $\left(4^{\circ} \mathrm{C}, 24^{\circ} \mathrm{C}\right.$ and $37^{\circ} \mathrm{C}$ ). The storage stability indicators (A) particle sizing and (B) \% encapsulation efficiency were determined at start of the study and at the end of 3 month storage period. All the samples were taken in triplicates. The data were plotted as mean \pm SEM. 
A
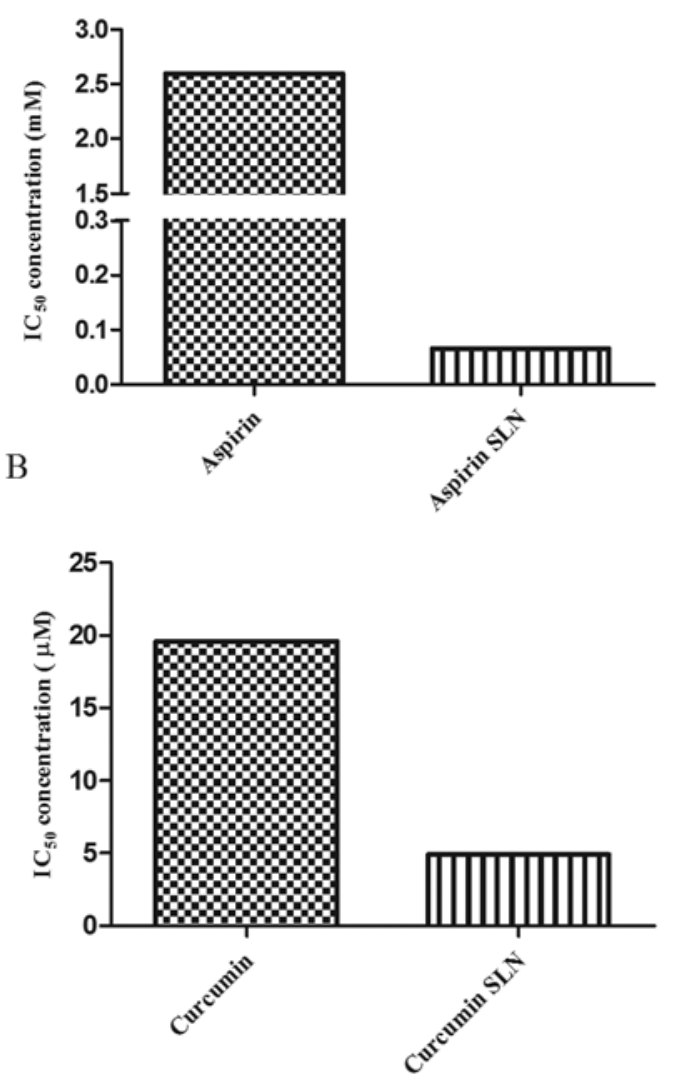

$\mathrm{C}$
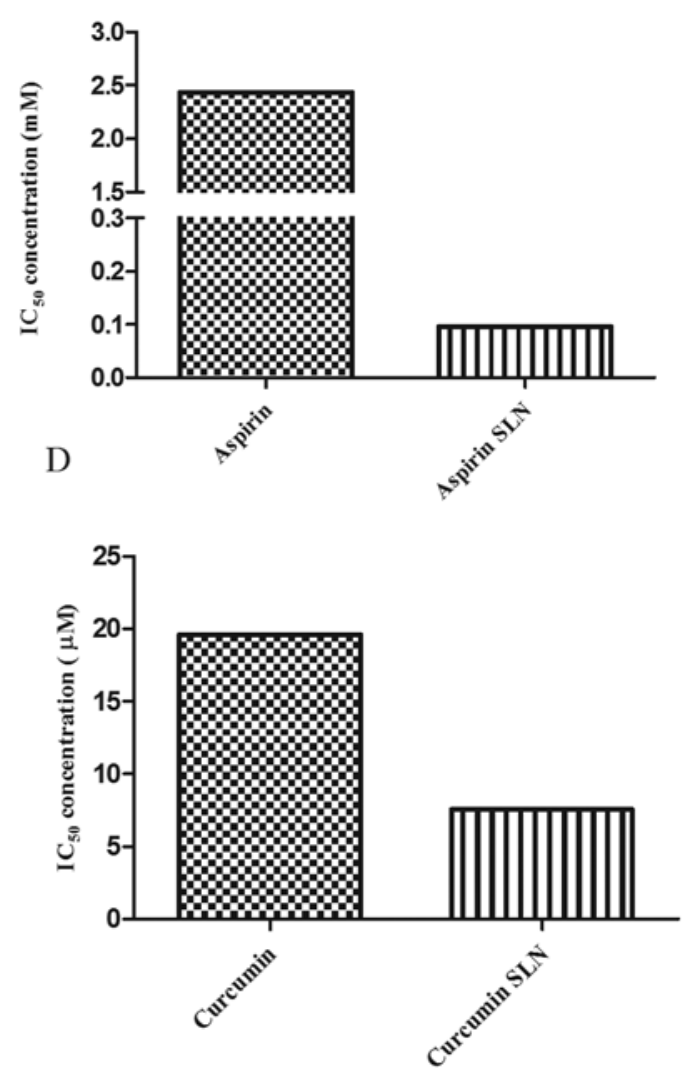

Figure 3. $\mathrm{IC}_{50}$ values comparison between free form and drug encapsulated solid lipid nanoparticles (SLNs). In MIA PaCa-2 cells, IC $\mathrm{C}_{50}$ values were compared between free and SLN forms of (A) aspirin and (B) curcumin. In Panc-1 cells, $\mathrm{IC}_{50}$ comparisons were shown between free and SLN forms of (C) aspirin and (D) curcumin. MTS assay was performed to determine the cell viability of MIA PaCa-2 and Panc-1 cells after treating with a range of concentrations of free and SLN forms of aspirin and curcumin for $72 \mathrm{~h}$. $\mathrm{IC}_{50}$ values were then determined using graphpad Prism software.

A

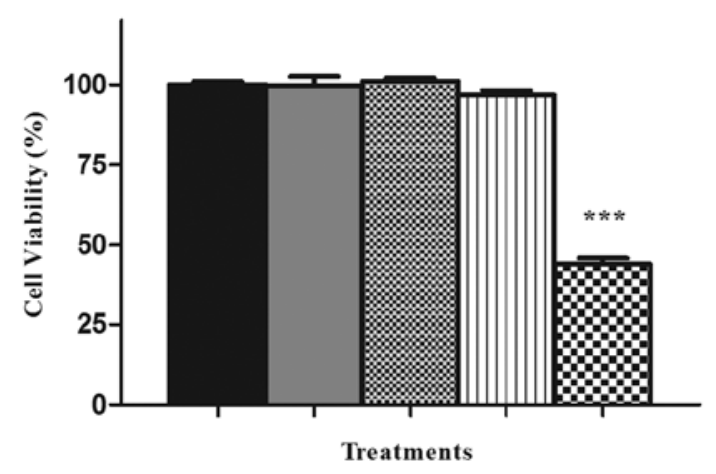

B

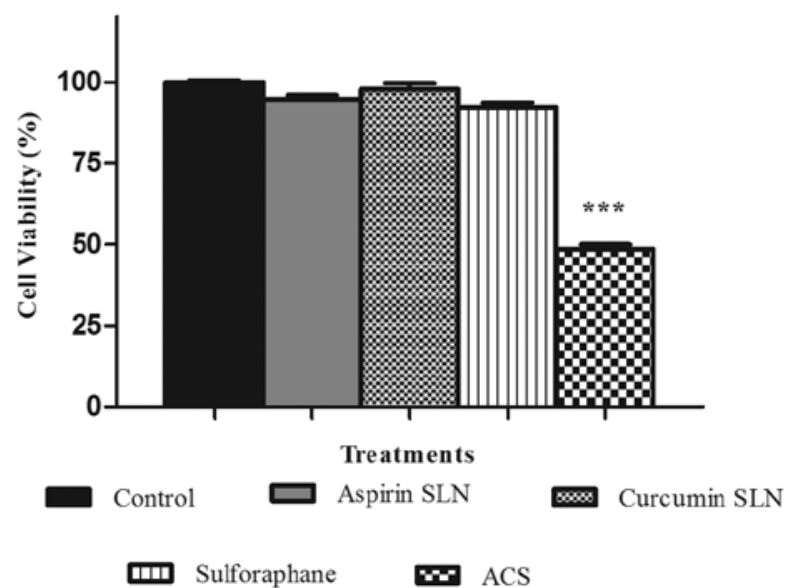

Figure 4. Synergistic effect of ACS combination on cell viability. MTS assay was performed to determine the cell viability of (A) MIA PaCa-2 and (B) Panc-1 cells after the treatment with aspirin SLN (ASP; $25 \mu \mathrm{M}$ ), curcumin SLN (CUR; $2.5 \mu \mathrm{M}$ ) and free sulforaphane (SFN; $5 \mu \mathrm{M}$ ) individually and in combination of ACS $(\mathrm{ASP}+\mathrm{CUR}+\mathrm{SFN}$ ) for $72 \mathrm{~h}$. Each bar represents the mean percent viable cells measured in three parallel but independent experiments. Statistical significance was determined by one-way ANOVA followed by Tukey's post hoc analysis. ${ }^{* * *} \mathrm{P}<0.001$ represents statistical significance of differences between control and treatment group.

were $19.6 \mu \mathrm{M}$ and $7.569 \mu \mathrm{M}$, respectively (Fig. 3D). Thus, our results demonstrate that the drugs when encapsulated in SLNs exhibited cytotoxicity at lower concentrations compared to free forms of the drugs. 
Aspirin SLN

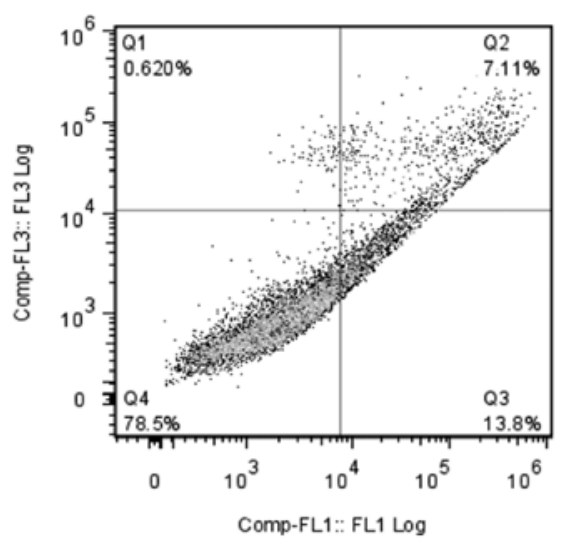

Sulforaphane

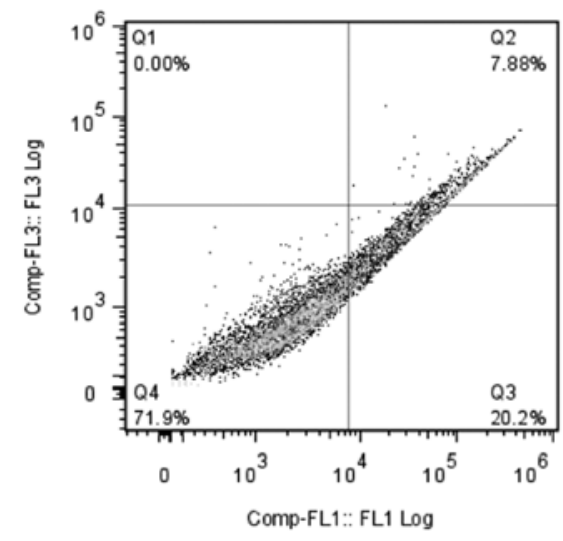

Curcumin SLN

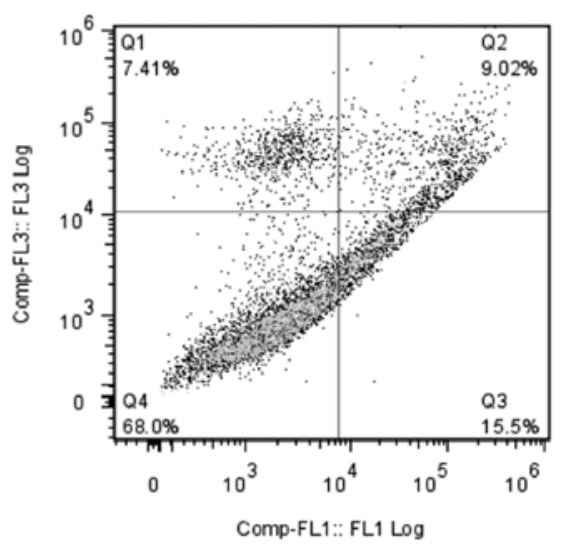

ACS

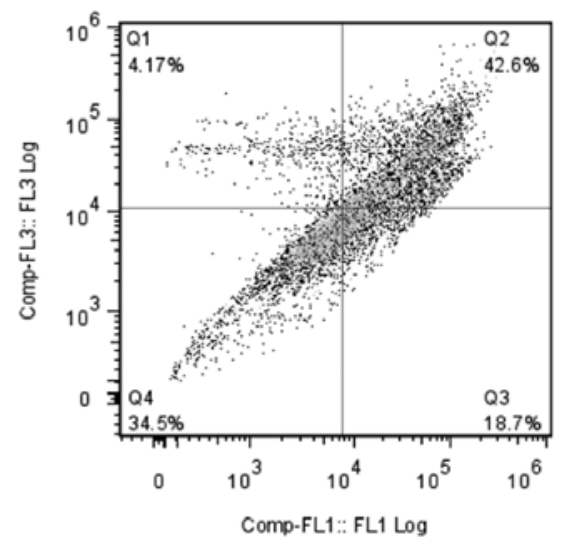

Figure 5. ACS combination induces apoptosis in pancreatic cancer cells. MIA PaCa-2 cells were treated with aspirin SLN (ASP; $25 \mu \mathrm{M}$ ), curcumin SLN (CUR; $2.5 \mu \mathrm{M})$ and free sulforaphane (SFN; $5 \mu \mathrm{M}$ ) or in combination ACS (ASP+CUR+SFN) for $48 \mathrm{~h}$. At the end of treatment, adherent and non-adherent cells were collected and stained with Annexin V-PI kit. Stained cells were then analyzed by flow cytometric analysis. The ACS chemopreventive combination demonstrated $\sim 61 \%$ apoptosis in MIA PaCa-2 cells.

Novel combined chemopreventive regimen of ASP SLNS, CUR SLNs and free SFN show a synergistic effect on the reduction of cell viability. MTS assay was performed in order to study the combination effects of chemopreventive drug SLNs on the cell lines MIA PaCa-2 and Panc-1. After determining the dose response curves individually and obtaining the $\mathrm{IC}_{50}$ value for each of them, ineffective drug concentrations were selected for ASP SLN $(25 \mu \mathrm{M})$, CUR SLN $(2.5 \mu \mathrm{M})$ showing minimal inhibitory response on the cell lines. SFN was used in its unmodified form at a concentration of $5 \mu \mathrm{M}$. When combined together as a combination (ACS), the cell viability was reduced to $43.6 \%$ for MIA PaCa-2 cell line (Fig. 4A) and $48.49 \%$ for Panc-1 cell line (Fig. 4B), respectively. This change was significant $(\mathrm{p}<0.0001)$ in comparison to the reduction in cell viability of individual concentrations of $\sim 10 \%$. Thus, synergistic effects were observed when combination of drugs was used. Dual combinations showed reduction in cell viability of $20 \%$ hence demonstrating no significant differences when compared to individual drug concentrations (data not shown).

Combined chemopreventive regimen shows increased apoptosis in human pancreatic cancer cells. Induction of apoptosis by $\operatorname{ASP}(25 \mu \mathrm{M}), \operatorname{CUR}(2.5 \mu \mathrm{M})$ and SFN $(5 \mu \mathrm{M})$ alone or the combination was evaluated in MIA PaCa- 2 and Panc- 1 cells. The cells were exposed to the drugs for $48 \mathrm{~h}$, and induction of cell apoptosis was examined by Annexin-V binding and PI staining assay. In case of MIA PaCa- 2 cell line as shown by the representative contour plots (Fig. 5), individual concentrations of SLN chemopreventive agents showed low evidence of apoptosis in both the cell lines; ASP SLNs exhibited $20.91 \%$, CUR SLNs $24.52 \%$ and SFN $28.08 \%$ apoptotic cells. However, when the cells were exposed to a combination (ACS), an apoptotic effect of $61.3 \%$ was observed.

A similar effect was observed in the case of Panc-1 cells (Fig. 6) where individual drug concentrations of ASP SLNs, CUR SLNs and SFN exhibited $8.84 \%, 12.14 \%$ and $16.55 \%$ of apoptotic cells, respectively. When the combination was used, increase in apoptotic effect was observed showing $60.37 \%$ apoptotic cells. Thus, the combination of SLN chemopreventive agents was synergistically effective in inducing apoptosis at low concentrations in both MIA PaCa-2 and Panc-1 cells.

\section{Discussion}

Pancreatic cancer ranks as the fourth most deadly form of cancer in the United States with approximately 37,000 deaths 
Aspirin SLN

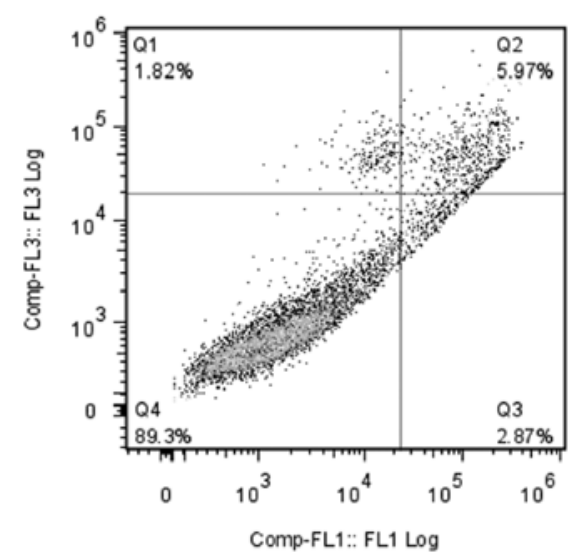

Sulforaphane

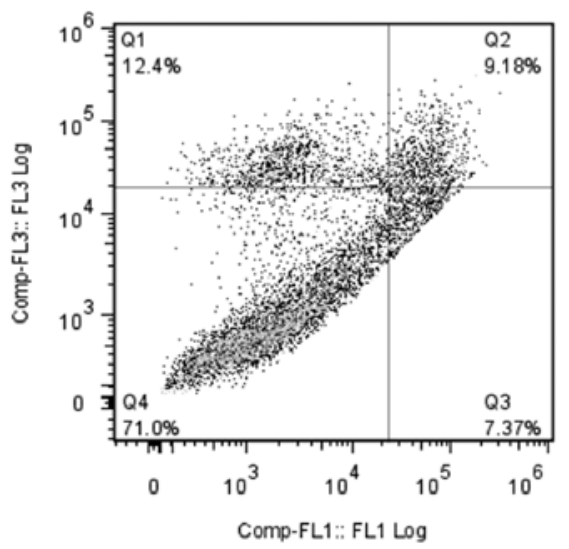

Curcumin SLN

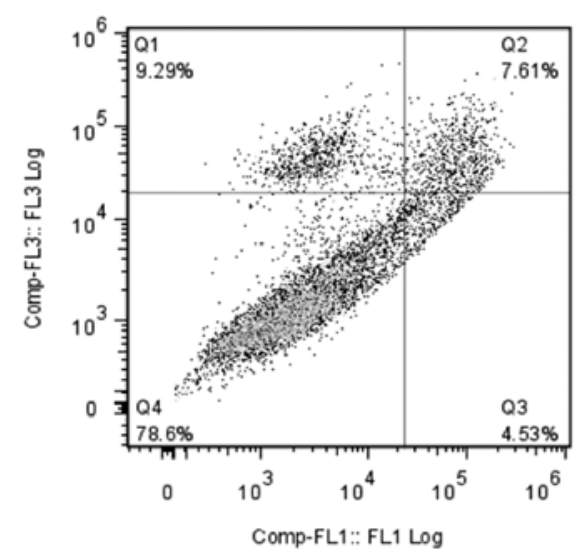

ACS

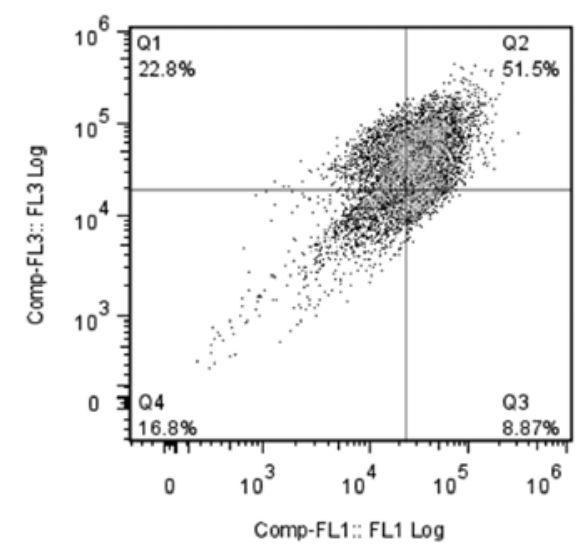

Figure 6. ACS combination induces apoptosis in pancreatic cancer cells. Panc-1 cells were treated with aspirin SLN (ASP; $25 \mu \mathrm{M}$ ), curcumin SLN (CUR; $2.5 \mu \mathrm{M}$ ) and free sulforaphane (SFN; $5 \mu \mathrm{M}$ ) or in combination ACS (ASP+CUR+SFN) for $48 \mathrm{~h}$. At the end of treatment, adherent and non-adherent cells were collected and stained with Annexin V-PI kit. Stained cells were then analyzed by flow cytometric analysis. The ACS chemopreventive combination demonstrated $\sim 60 \%$ apoptosis in Panc-1 cells.

each year (1). Early diagnosis of this disease is difficult because it develops without any early symptoms. Survival of patients with pancreatic cancer has been $<5 \%$ over 5 years which makes this disease of great concern (2). Therapeutic outcomes with pancreatic cancer are not useful for patients especially upon a late diagnosis thus strategies to prevent this disease from occurring have become an important area of research. Recently, chemoprevention has emerged as an effective tool in the fight against various types of cancers, including colon and pancreatic cancer $(19,27)$.

Our research has been focused on the administration of low doses of ACS combination SLNs to study its chemopreventive effects against pancreatic cancer cells MIA PaCa-2 and Panc-1. Single agent administration at low concentrations has been demonstrated to be ineffective, hence the hypothesis that two or more chemopreventive agents when delivered at low concentrations together, may exhibit an additive or synergistic effect against the cancer cells was tested. This can be attributed to the multi-factorial nature of carcinogenesis wherein cancer occurs as a result of multiple cellular changes during a prolonged time period. Of the various chemopreventive agents being studied, ASP, CUR and SFN have been proven to be effective in the chemoprevention of pancreatic cancer (2-4). Several in vivo and in vitro studies have shown that NSAIDs like aspirin and celecoxib have helped prevent the progression of pancreatic cancer. CUR and SFN, both being effective and non-toxic in nature, have been investigated for their chemopreventive actions $(9,12,17)$. Therefore using a multi-disciplinary approach, this study investigated the synergistic effects of SLN combinations of chemopreventive agents, namely, ASP in combination with CUR and SFN.

Nevertheless, the clinical translation of these agents has been significantly hampered due to various reasons including poor oral bioavailability after administration. Engineering an SLN drug delivery system for these agents offers a means of increasing the bioavailability in the plasma and tissues in comparison to the free form of these drugs, thereby ultimately improving the therapeutic efficacy. Also, encapsulating the drug within the lipid matrix allows for administration of lower doses of drugs with less chance of toxic effects, while maintaining efficacy. Furthermore, using combination of agents which differ in their mode of action helps to obtain a desired preventive effect, while minimizing the dose concentration and its toxic effects (26). In terms of formulation development, both ASP and CUR SLNs were found to have optimal particle sizes, high encapsulation efficiency with good stability at or below room 
temperature. The improved stability may be explained by the use of organic solvent in the preparation process which may have improved the hygroscopic nature of the lipid. The high encapsulation can be attributed to the lipophilic nature of both ASP and CUR. Also the particle sizing was found to be in nanometer range suggesting a better chance of cellular uptake of the drugs. The formulated SLNs are non-toxic because they are made of physiological lipids such as stearic acid.

The effect of these agents was initially evaluated by calculating the $\mathrm{IC}_{50}$ values and then by combining the ineffective concentrations to exhibit an additive or synergistic effect against the cancer cells proving to be more efficacious at lower concentrations. A comparative study between the two forms of the drugs i.e., the free form and SLN form was carried out. It was observed that ASP and CUR SLNs $\mathrm{IC}_{50}$ concentrations exhibited approximately 38- and 3-fold reductions, respectively in comparison to the free form of the drug. Studies have been reported where drug loaded SLNs have exhibited better cytotoxicity profile in comparison to the free drug (28). This has been mainly attributed to the smaller particle size of the nanoparticles which increases the overall uptake of the drug. The surfactant used in the development process determines the inhibitory effect on the cells. We used Poloxamer 188 which has previously been shown to target cancerous cells, due to differences in the membrane of these cells when compared to the non-cancer cells. Poloxamer has not only been shown to inhibit multiple drug resistance (MDR) proteins and other drug efflux transporters on the surface of the cancer cells but also shown to enhance protoapoptotic signaling and decrease anti-apoptotic defense in MDR cells (29). The MTS assay on drug entrapped SLNs was carried out using ASP $(25 \mu \mathrm{M})$, CUR $(2.5 \mu \mathrm{M})$ and SFN $(5 \mu \mathrm{M})$ as individual concentrations. Individually they showed little or no decrease in the cell viability in the two cell lines, but when combined, a significant reduction by $60 \%$ was observed in MIA PaCa-2 and Panc-1 cells. In order to validate the efficacy of the combination regimen, apoptosis assay was conducted which determined the progression of a cancer cell from four different phases after the addition of the drug; living cell, early apoptotic cell, late apoptotic cell and necrotic cells. These results seem to be consistent with our findings in the MTS assay. The possible mechanisms involving the significant change for the combination could be an additive effect of the COX-2 enzyme inhibition, the regulation of the P53 suppressor pathway and by the modulation of the Nrf2 pathway; however additional studies need to be done to verify the findings.

From these results, we believe that chemoprevention is an effective way to prevent pancreatic cancer especially as the disease cannot be diagnosed at an early stage. Using a multidisciplinary approach, this study investigated the synergistic effects of a combination of ASP and CUR SLN with free SFN. We demonstrated for the first time that this SLN combination showed a synergistic inhibition of cell viability and induce apoptosis in human pancreatic cancer cell lines. However, further in vivo studies have to be conducted to test the efficacy of this SLN combination. In conclusion, the results obtained from formulation studies and cell based assays clearly demonstrate the scope of developing the combination drug encapsulated SLN formulations to prevent pancreatic cancer.

\section{Acknowledgements}

This study was supported by a grant from National Institutes of Health (1R03CA153812-01A1; SP).

\section{References}

1. Siegel R, Naishadham D and Jemal A: Cancer statistics, 2012. CA Cancer J Clin 62: 10-29, 2012.

2. Fendrich V: Chemoprevention of pancreatic cancer-one step closer. Langenbecks Arch Surg 397: 495-505, 2012.

3. Logsdon CD and Abbruzzese JL: Chemoprevention of pancreatic cancer: ready for the clinic? Cancer Prev Res (Phila) 3: 1375-1378, 2010.

4. Husain SS, Szabo IL and Tamawski AS: NSAID inhibition of GI cancer growth: clinical implications and molecular mechanisms of action. Am J Gastroenterol 97: 542-553, 2002.

5. Chan AT, Giovannucci EL, Schernhammer ES, et al: A prospective study of aspirin use and the risk for colorectal adenoma. Ann Intern Med 140: 157-166, 2004.

6. Kokawa A, Kondo H, Gotoda T, et al: Increased expression of cyclooxygenase- 2 in human pancreatic neoplasms and potential for chemoprevention by cyclooxygenase inhibitors. Cancer 91: 333-338, 2001.

7. Jacobs EJ, Connell CJ, Rodriguez C, Patel AV, Calle EE and Thun MJ: Aspirin use and pancreatic cancer mortality in a large United States cohort. J Natl Cancer Inst 96: 524-528, 2004.

8. Stan SD, Singh SV and Brand RE: Chemoprevention strategies for pancreatic cancer. Nat Rev Gastroenterol Hepatol 7: 347-356, 2010.

9. Kuo ML, Huang TS and Lin JK: Curcumin, an antioxidant and anti-tumor promoter, induces apoptosis in human leukemia cells. Biochim Biophys Acta 1317: 95-100, 1996.

10. Sarkar FH, Banerjee S and Li Y: Pancreatic cancer: pathogenesis, prevention and treatment. Toxicol Appl Pharmacol 224: 326-336, 2007.

11. Sa G and Das T: Anti cancer effects of curcumin: cycle of life and death. Cell Div 3: 14, 2008.

12. Moragoda L, Jaszewski R and Majumdar AP: Curcumin induced modulation of cell cycle and apoptosis in gastric and colon cancer cells. Anticancer Res 21: 873-878, 2001.

13. Li DW, Liu JP, Mao YW, et al: Calcium-activated RAF/MEK/ ERK signaling pathway mediates p53-dependent apoptosis and is abrogated by alpha B-crystallin through inhibition of RAS activation. Mol Biol Cell 16: 4437-4453, 2005.

14. Romashkova JA and Makarov SS: NF-kappaB is a target of AKT in anti-apoptotic PDGF signalling. Nature 401: 86-90, 1999.

15. Matusheski NV, Juvik JA and Jeffery EH: Heating decreases epithiospecifier protein activity and increases sulforaphane formation in broccoli. Phytochemistry 65: 1273-1281, 2004.

16. Kallifatidis G, Rausch V, Baumann B, et al: Sulforaphane targets pancreatic tumour-initiating cells by NF-kappaB-induced antiapoptotic signalling. Gut 58: 949-963, 2009.

17. Lampe JW: Sulforaphane: from chemoprevention to pancreatic cancer treatment? Gut 58: 900-902, 2009.

18. Rao CV, Newmark HL and Reddy BS: Chemopreventive effect of farnesol and lanosterol on colon carcinogenesis. Cancer Detect Prev 26: 419-425, 2002.

19. Zhou P, Cheng SW, Yang R, Wang B and Liu J: Combination chemoprevention: future direction of colorectal cancer prevention. Eur J Cancer Prev 21: 231-240, 2012.

20. Chaudhary A, Wang J and Prabhu S: Development and validation of a high-performance liquid chromatography method for the simultaneous determination of aspirin and folic acid from nanoparticulate systems. Biomed Chromatogr 24: 919-925, 2010.

21. Prabhu S, Tran LP and Betageri GV: Effect of co-solvents on the controlled release of calcitonin polypeptide from in situ biodegradable polymer implants. Drug Deliv 12: 393-398, 2005.

22. Brannon-Peppas L and Blanchette JO: Nanoparticle and targeted systems for cancer therapy. Adv Drug Deliv Rev 56: 1649-1659, 2004.

23. Müller RH, Mäder K and Gohla S: Solid lipid nanoparticles (SLN) for controlled drug delivery - a review of the state of the art. Eur J Pharm Biopharm 50: 161-177, 2000.

24. Uner $M$ and Yener G: Importance of solid lipid nanoparticles (SLN) in various administration routes and future perspectives. Int J Nanomed 2: 289-300, 2007. 
25. Yuan H, Chen J, Du YZ, Hu FQ, Zeng S and Zhao HL: Studies on oral absorption of stearic acid SLN by a novel fluorometric method. Colloids Surf B Biointerfaces 58: 157-164, 2007.

26. Chaudhary A, Sutaria D, Huang Y, Wang J and Prabhu S Chemoprevention of colon cancer in a rat carcinogenesis model using a novel nanotechnology-based combined treatment system. Cancer Prev Res (Phila) 4: 1655-1664, 2011.

27. Reddy BS, Nayini J, Tokumo K, Rigotty J, Zang E and Kelloff G: Chemoprevention of colon carcinogenesis by concurrent administration of piroxicam, a nonsteroidal antiinflammatory drug with D,L-alpha-difluoromethylornithine, an ornithine decarboxylase inhibitor, in diet. Cancer Res 50: 2562-2568, 1990.
28. Miglietta A, Cavalli R, Bocca C, Gabriel L and Gasco MR: Cellular uptake and cytotoxicity of solid lipid nanospheres (SLN) incorporating doxorubicin or paclitaxel. Int J Pharm 210: 61-67, 2000.

29. Yan F, Zhang C, Zheng Y, et al: The effect of poloxamer 188 on nanoparticle morphology, size, cancer cell uptake, and cytotoxicity. Nanomedicine 6: 170-178, 2010. 